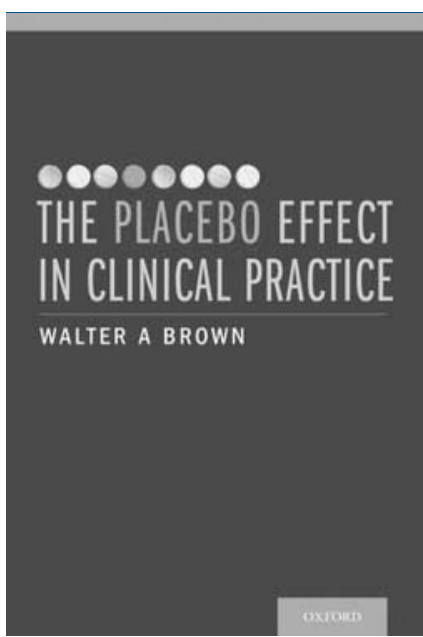

\section{The Placebo Effect}

\section{in Clinical Practice}

By Walter A Brown.

Oxford University Press. 2013.

£30.00 (hb). $177 \mathrm{pp}$.

ISBN: 9780199933853
The placebo effect phenomenon has pervaded modern medical practice for some time and is subject to much debate over both its necessity in patient treatment and its ethical implications. These issues are successfully raised in a book that is well structured and covers the topic with great breadth and depth. The concept of the placebo effect is successfully introduced; the opening chapter draws on what we know historically about placebo and its use in current practice. The book focuses on the use of placebo not only in psychiatry but also in other specialties - anaesthesia, gastroenterology and cardiology. In doing so, the reader is able to appreciate the variation of placebo effects across the board and its comparability to active treatment in terms of patient outcomes.

Professor Brown successfully tackles the cynic's view that placebo is no different from receiving no treatment at all. Great emphasis is placed on not seeing the placebo as a sole entity but acknowledging the 'treatment situation' within which it is delivered, the manner in which this is done by the professional and the expectations of the patient. Several theories are ascribed to placebo and a chapter is dedicated to the conditioning theory and its perceived integral role in the whole process. Professor Brown puts forward a convincing argument for the deliberate use of placebo within daily clinical practice, but there is still an appreciation that placebo may not be suitable in all circumstances. Objections to the use of placebo are foreseen and carefully explored.

In summary, this book provides a balanced overview of the placebo response in clinical practice that sheds some light and clarity for the reader on what can otherwise be perceived as a complex phenomenon.

Sarah Jawad CT3 psychiatry, Birmingham and Solihull Mental Health NHS Foundation Trust, The Barberry, 25 Vincent Drive, Edgbaston, Birmingham B15 2FG, UK. Email: sarah.jawad@bsmhft.nhs.uk

doi: 10.1192/bjp.bp.113.131771 